\section{Cahiers de Narratologie}

Analyse et théorie narratives

$14 \mid 2008$

Prose d'idées : formes et savoirs

\title{
Mythe et violence : Georges Sorel dans les manifestes du Futurisme italien
}

\section{Serge Milan}

\section{(2) OpenEdition}

\section{Journals}

Édition électronique

URL : http://journals.openedition.org/narratologie/634

DOI : 10.4000/narratologie.634

ISSN : 1765-307X

Éditeur

LIRCES

Référence électronique

Serge Milan, «Mythe et violence : Georges Sorel dans les manifestes du Futurisme italien », Cahiers de Narratologie [En ligne], 14 | 2008, mis en ligne le 06 mars 2008, consulté le 15 novembre 2019. URL : http://journals.openedition.org/narratologie/634 ; DOI : 10.4000/narratologie.634

Ce document a été généré automatiquement le 15 novembre 2019.

\section{(c) (i) (9)}

Cahiers de Narratologie - Analyse et théorie narratives est mis à disposition selon les termes de la licence Creative Commons Attribution - Pas d'Utilisation Commerciale - Pas de Modification 4.0 International. 


\title{
Mythe et violence : Georges Sorel dans les manifestes du Futurisme italien
}

\author{
Serge Milan
}

1 Le phénomène avant-gardiste, qui caractérise sans aucun doute l'esthétique du $\mathrm{XX}^{\mathrm{e}}$ siècle plus que tout autre, doit être appréhendé également comme une tentative fortement structurée de production idéologique à vaste échelle.

2 A cet égard, par-delà les spectacles ou les œuvres plastiques et littéraires produites par les différentes avant-gardes, l'avant-gardisme est lié de façon inhérente à la production d'une catégorie de textes qu'on peut définir comme "prose d'idées", et dont les articles, essais, proclamations, manifestes et tracts divers constituent un corpus hétérogène et foisonnant.

3 La prose d'idée avant-gardiste, en plus de caractéristiques formelles et stylistiques tout à fait originales - comme son rapport aux œuvres plastiques, sa dimension polémique et normative, ou encore l'utilisation du manifeste comme un véritable fer de lance propagandiste - se distingue par un rapport aux textes philosophiques assez remarquable. En effet, en plus de la vulgarisation inhérente sans doute à la prose d'idée dans son ensemble, l'un des principaux traits que nous souhaitions illustrer ci-dessous consiste en une auto-illustration à la fois pragmatique et opératoire des textes, qui renvoie très directement aux projets politiques et anthropologiques des avant-gardes.

Dès lors, il s'agit de revenir sur les sources idéologiques du Futurisme italien, qui peut être considéré comme la première avant-garde pluridisciplinaire et dont le projet, démiurgique et totalisant, inclut une anthropogenèse originale et radicale. En examinant les œuvres et en relisant les manifestes du mouvement (1909-1944), plusieurs chercheurs ont déjà indiqué certains des inspirateurs de ces poètes et artistes comme Bergson et Nietzsche ${ }^{1}$. Nous souhaitions nous arrêter ici sur l'influence de Georges Sorel (1847-1922), ce marxiste atypique qui a marqué profondément la pensée politique française et italienne du début $\mathrm{XX}^{\mathrm{e}}$ siècle, et notamment l'anarchosyndicalisme. Son volume de 1908 Réflexions sur la violence a en effet été cité pour ce qui 
concerne la polémologie futuriste, mais également et plus précisément pour une apologie de la violence physique qui sera pratiquée par beaucoup d'avant-gardistes et d'activistes politiques : il faut préciser ici quels textes et quelles notions sont à relier dans cette filiation idéologique, mais également en examiner les modalités intertextuelles.

$5 \quad$ Les Réflexions sur la violence et leur rapport au Futurisme peuvent être examinées plus précisément à partir de quelques manifestes en particulier, tous signés par le fondateur et l'organisateur de cette avant-garde, Filippo Tommaso Marinetti. Il s'agit essentiellement de 1915 In quest'anno futurista, publié fin 1914, mais également d'autres textes comme Al di là del Comunismo, publié en 1920. Ce sont là des manifestes fort représentatifs de l'engagement du mouvement futuriste et de sa conception du politique, par lesquels cette avant-garde exprime pleinement sa volonté de peser dans l'élaboration de la jeune société italienne non seulement d'un point de vue artistique et culturel, mais plus profondément par des choix idéologiques fondateurs d'une Italie débarrassée de tout passéisme, pour reprendre l'une des notions centrales du mouvement, complexe et polysémique.

" 1915 En cette année futuriste »

61915 In quest'anno futurista, comme quelques autres textes futuristes d'importance, sera publié plusieurs fois dans les revues futuristes ou dans des recueils de textes du mouvement : après une première publication in Guerra sola igiene del mondo, pour les Editions futuristes de Poesia à Milan, en 1915, il sera ainsi republié par exemple en 1919 dans le recueil I manifesti del Futurismo, Istituto Editoriale Italiano, toujours à Milan, et en 1924 dans Futurismo e fascismo, Franco Campitelli Editore, Foligno. Par ailleurs, ce manifeste, que nous nous proposons de considérer à l'aune des manifestes futuristes comme une œuvre littéraire à part entière, sera également publié sous forme d'article dans plusieurs revues, dont l'une des revues les plus marquantes de l'avant-garde italienne des années Dix, L'Italia Futurista ${ }^{2}$.

Ce texte synthétise parfaitement les caractéristiques des manifestes définis non par leur sectorialité (comme par exemple le Manifesto della pittura futurista, Il Teatro del varietà, La fotografia futurista ou plusieurs autres dizaines de manifestes édictant de nouvelles règles pour chaque art ou discipline examinée individuellement), ni $a$ fortiori par leur visée technique, comme le Manifesto tecnico della letteratura futurista, mais au contraire par leur absence d'implication esthétique sectorielle directe: ce sont alors plutôt les destinataires qui induisent son contenu - dans ce cas, les étudiants italiens, apostrophés à plusieurs reprises et auxquels était explicitement destiné le manifeste, intitulé précisément Manifesto agli studenti dans sa version de 1924.

8 Les étudiants, donc : c'est-à-dire avant tout les jeunes, dont le Futurisme avait dès sa fondation fait la principale cible de sa propagande et qui constituaient l'essentiel de ses adhérents, poètes, écrivains, peintres, graphistes, architectes, scénographes ou autres ; mais alors qu'il s'agissait dans le manifeste de fondation de 1909 d'interpeller les créateurs, le champ s'ouvre ici davantage encore sur le modèle du premier manifeste politique de 1909, qui invoquait comme électeurs futuristes "tous les jeunes esprits d'Italie"3. Cet appel à la jeunesse, initialement très polémique à l'égard des aînés (il s'agissait de "combattre brutalement les vieux" dans une "lutte à outrance"4) est repris par les peintres en 1910 dans un slogan à succès qui résumera en partie les aspirations culturelles et politiques de l'avant-garde et de la contre-culture du siècle: Largo ai giovani ! 
9 Cette apologie de la jeunesse iconoclaste et rebelle, qui ne se démentira jamais dans l'avant-garde, est par ailleurs ici indissociable d'un souci hygiéniste scientiste, notamment chez Marinetti. De même que la guerre est définie comme "la seule hygiène du monde" dès le premier manifeste, de même que la ville et la cuisine futuriste répondent avant tout à des critères fonctionnels d'hygiène ${ }^{6}$, de même le Futurisme luimême est "une école d'hygiène spirituelle, une sorte de jeunesse artificielle qui finit par devenir naturelle, et qui empêche de devenir plagiaire, précautionneux, diplomate" :

Le mot Futurisme contient la formule de renouvellement la plus vaste : celle qui, étant à la fois hygiénique et excitante, simplifie les doutes, détruit les scepticismes et réunit les efforts en une formidable exaltation. Tous les novateurs se rencontrent sous le drapeau du Futurisme, parce que le Futurisme proclame la nécessité d'aller toujours de l'avant, et parce qu'il propose la destruction de tous les ponts offerts à la lâcheté. Le Futurisme est l'optimisme artificiel opposé à tous les pessimismes chroniques, il est le dynamisme continu, le devenir perpétuel et la volonté infatigable. Le Futurisme n'est donc pas soumis aux lois de la mode ni à l'usure du temps, il n'est pas une chapelle ni une école, mais plutôt un grand mouvement solidaire d'héroïsmes intellectuels dans lequel l'individu n'est rien, tandis que la volonté de renouveler est tout. ${ }^{7}$

10 Cette définition, tirée précisément de 1915 In quest'anno futurista, est extraite d'un long passage, sans doute l'une des tentatives les plus complètes cherchant à définir lyriquement et conceptuellement le mouvement; en plus de proposer à nouveau "le mot Futurisme" comme terme emblématique et autoréférentiel, ce paragraphe revient sur la visée éthique de l'avant-garde en insistant une fois de plus sur sa vertu curative, rajeunissante et militarisante.

11 La technique marinettienne de propagande est ici particulièrement efficace ${ }^{8}$ : l'introduction construite autour de la métaphore médicale souligne ce que les futuristes eux-mêmes appelleront le caractère "anhistorique" de leur mouvement et sa valeur supranationale, tout en faisant de l'Italie précisément un point de départ historique privilégié de ce renouveau universel ("L'Italie, plus que tout autre pays, avait un besoin urgent de Futurisme, car elle mourait de passéisme. Le malade inventa son propre remède. Nous sommes ses médecins occasionnels. Le remède vaut pour les malades de tous les pays"9).

12 À partir de cette image thaumaturgique, le Futurisme devient dans le premier tiers du manifeste un objet poétique et conceptuel fascinant, surdéterminé par une accumulation de définitions mais simultanément toujours masqué par des métaphores incessantes, hétéroclites et baroques ; une partie centrale, isolée typographiquement, fonctionne comme arsenal d'arguments polémiques et rhétoriques, tandis que la dernière partie est une apologie de la Guerre élevée à principe ontologique, comme une prolongation spéculaire du Futurisme défini auparavant.

Polémologie et propagande

13 On retrouve par ailleurs dans ce texte la plupart des techniques propagandistes marinettiennes, de l'autocitation à la typographie expressive, avec également une liste des principaux artistes du mouvement, l'invention de slogans et de mots d'ordre percutants, la réponse polémique à la réinterprétation brutale du passé poétique européen, et enfin le rappel des inventions futuristes, ainsi qu'une synthèse finale survoltée, appel à la mobilisation en masse, à la substitution des figures intellectuelles paternelles par un modèle éthique identificatoire (lo scugnizzo, c'est-à-dire le gamin voyou des rues napolitaines) et la désignation des coupables de neutralisme passéiste, 
nommément cités. Il faut encore rappeler que 1915 In quest'anno futurista est repris en partie d'un article de 1913 et qu'il sera encore repris, quasiment tel quel, dans un autre ouvrage de 1919, donnant lieu à une multiplication de republications et à un recyclage textuel caractéristiques de la propagande de Marinetti ${ }^{10}$.

Le paragraphe central de la première partie, mettant en cause les écrivains "semi futuristes" ou "mal convertis au Futurisme", ne peut être interprété que comme une attaque à Giovanni Papini et à ses amis, Ardengo Soffici en tête ${ }^{11}$, après les tensions entre ces avant-gardistes florentins de la revue Lacerba et la Direction milanaise du mouvement constituée pour l'essentiel de Marinetti lui-même et du peintre et sculpteur Umberto Boccioni. Après ces piques, la reprise des définitions du Futurisme doit donc se lire également de façon discriminatoire : Marinetti se démarque des "semi futuristes" en les renvoyant ainsi à un nihilisme dont il n'a jamais aussi clairement réfuté les limites. "Nous sommes des entrepreneurs de démolition, mais pour reconstruire", écrit-il, reprenant très synthétiquement un thème qu'on peut interpréter comme une confirmation supplémentaire et à certains égards définitive du dépassement du post-symbolisme qui imprégnait initialement le mouvement.

Pour ce qui est du manifeste, les choix stylistiques illustrent finalement ce qu'on pourrait appeler la stratégie incantatoire de Marinetti, qui parvient parfaitement à dépasser toutes les contingences autour du mouvement pour aboutir à une compréhension si vaste du terme qu'il en devient quasiment autoréférentiel, le mot Futurisme étant mis en abyme ("la parola Futurismo") et se substituant finalement à tous ceux qui avaient contribué à le définir imparfaitement. Cela est confirmé à la fin de la partie centrale du texte :

Les foules qui nous ont sifflés ont involontairement admiré en nous des artistes désintéressés qui luttent hérö̈quement afin de rajeunir, renforcer et accélérer le génie italien. Le grand bloc d'idées nouvelles que nous avons formé roule ici et là dans la boue et sur les pierres, poussé et sali par les mains de joyeux chenapans. Ceux-ci, se moquant des étranges couleurs apparentes de cet énorme jouet inattendu, subissent son contenu incandescent et magnétique. Ce n'est pas de la rhétorique : le mot Futurisme a fait, prodigieusement et à lui seul, beaucoup de bien à l'Italie et au monde. Partout et à tout propos, dans les parlements, les conseils municipaux et sur les places, les hommes se divisent en passéistes et futuristes. (Aujourd'hui, en Italie, passéistes est synonyme de neutralistes, pacifistes et eunuques, tandis que futuristes est synonyme d'antineutralistes violents.) ${ }^{12}$

16 Nous reviendrons sur la proximité de ces dernières lignes avec celles des écrits de Sorel, qui font de ce manifeste un texte représentatif de la filiation que nous souhaitions illustrer. Mais la dernière partie de 1915 In quest'anno futurista mérite ici une attention toute particulière, en ce qu'elle représente un aboutissement pour ce qui est de la polémologie futuriste. Il s'agit d'une des thématiques les plus récurrentes et controversées du mouvement, dont les développements semblent omniprésents tant dans l'idéologie que dans les œuvres de cette avant-garde.

Cette polémologie se développe d'abord essentiellement dans un cadre gnoséologique, dans cette volonté première d'un rejet brutal du passé, de ses institutions et de ses valeurs; à partir de 1911, pourtant, et jusque dans la polémique contre Lacerba, Marinetti insistera sur la signification politique et patriotique du point $n^{\circ} 9 \mathrm{du}$ manifeste de fondation ("Nous voulons glorifier la guerre - seule hygiène du monde - le militarisme, le patriotisme, le geste destructeur des libertaires, les belles idées pour lesquelles on meurt et le mépris de la femme") : après la Grande Guerre et tout au long 
du Ventennio, il n'aura de cesse de rappeler les manifestations anti-autrichiennes des débuts du mouvement qui lui valurent, ainsi qu'à ses camarades futuristes, des arrestations répétées ${ }^{13}$.

De façon fort surprenante en cette veillée d'armes, 1915 In quest'anno futurista reprend pourtant la thématique polémologique en la recentrant directement sur la guerre plutôt que sur un ennemi désigné : certes, cela est cohérent avec des intuitions déjà présentes dans des textes écrits très tôt par Marinetti ("... il faut que l'âme lance le corps en flamme, comme un brûlot, contre l'ennemi, l'éternel ennemi qu'il faudrait inventer s'il n'existait pas !"14). Si l'Autriche est bien citée deux fois dans le manifeste, et si les allusions nationalistes sont plus nombreuses, force est de constater que la guerre est envisagée avant tout dans une signification bien plus large, investissant, tout comme le Futurisme dans la première partie du texte, les domaines bien plus hétérogènes, pour permettre encore une fois une multiplication surdéterminante de définitions et de métaphores. Le passage de relais entre les deux termes est souligné par le début de cette partie finale :

Étudiants italiens!

Le Futurisme dynamique et agressif se réalise aujourd'hui pleinement dans la grande guerre mondiale qu'il prévit et glorifia - seul - avant qu'elle n'éclate. La guerre actuelle est le plus beau poème futuriste apparu jusqu'ici : le Futurisme marqua précisément l'irruption de la guerre dans l'art, en créant ce phénomène qu'est la Soirée futuriste (propagande très efficace de courage). Le Futurisme fut la militarisation des artistes novateurs. Aujourd'hui, nous assistons à une immense exposition futuriste de tableaux dynamiques et agressifs, dans laquelle nous voulons très vite entrer et nous exposer..$^{15}$

Nous sommes revenus par ailleurs sur le commentaire que fait Walter Benjamin de ces propos dans un passage connu de L'œuvre d'art à l'ère de sa reproductibilité technique ${ }^{16}$, et notamment sur cette esthétisation de la guerre marinettienne qui changera de nature dans les années Trente et finira par muer en propagande mussolinienne. Cependant, c'est une polémologie plus idéale et plus radicale dont il est ici question: dans ce manifeste, il est remarquable que la guerre se substitue progressivement au Futurisme en tant que terme iconique autoréférentiel. Le passage ci-dessus est le dernier centré sur le Futurisme, sur sa définition sans cesse répétée et sur son anaphore hypnotique. C'est la guerre qui sera désormais objet d'un travail rhétorique très similaire, à commencer par un traitement typographique (majuscule systématique, caractères gras, signes algébriques) suggérant encore une fois l'hypostase. Après avoir vanté l'art futuriste guerrier et cité la plupart des artistes importants du mouvement, Marinetti s'en prend aux poètes passéistes :

Ces porte-paroles du pacifisme, en combattant l'Allemagne et l'Autriche, espèrent éliminer la Guerre, comme s'il s'agissait d'un reste de barbarie. La Guerre ne peut mourir, parce qu'elle est une loi de la vie. Vie = agression. Paix universelle = décrépitude et agonie des races. Guerre = essai sanglant et nécessaire de la force d'un peuple.

Ce qu'il faut éliminer, et ce qui doit mourir, c'est le passéisme teuton, fait de moutonnerie inintelligente, de balourdise pédante et professorale, d'obsession culturelle et plagiaire, d'orgueil paysan, d'espionnage systématique et d'imbécillité policière.

Nous autres mots-libristes, peintres, musiciens, bruiteurs et architectes futuristes avons toujours considéré la Guerre comme l'unique inspiration de l'art, l'unique morale salvatrice, l'unique levain de la pâte humaine. Seule la Guerre sait rajeunir, accélérer, aiguiser l'intelligence humaine, alléger et aérer les nerfs, nous libérer des poids quotidiens, donner mille saveurs à la vie et donner de l'esprit aux imbéciles. 
La Guerre est le seul gouvernail de plongée de la nouvelle vie aéroplanique que nous préparons.

La Guerre, Futurisme intensifié, ne tuera jamais la Guerre, comme l'espèrent les passéistes, mais elle tuera le passéisme. La Guerre est la synthèse culminante et parfaite du progrès (vitesse agressive + simplification violente des efforts vers le bien-être). La Guerre est une imposition foudroyante de courage, d'énergie et d'intelligence pour tous. École imposée d'ambition et d'héroïsme ; plénitude de vie et liberté maximale dans le dévouement à la patrie. ${ }^{17}$ à hauteur de principe vital : pourtant cette thèse, explicitée en deux phrases, dont une égalité arithmétique toute rhétorique ("La Guerre ne peut mourir, parce qu'elle est une loi de la vie. Vie = agression") n'est jamais approfondie, et elle semble même être limitée par son contexte au domaine politique (barbarie, razze, popolo). Il faut remarquer qu'on la retrouvait développée bien plus longuement chez Nietzsche, par exemple, chez qui elle prend, toujours à partir d'une problématique politique, une tout autre dimension :

... vivre, c'est essentiellement dépouiller, blesser, dominer ce qui est étranger et plus faible, l'opprimer, lui imposer durement sa propre forme, l'englober et au moins, au mieux, l'exploiter. [...]

[L'exploitation] est inhérente à la vie dont elle constitue une fonction primordiale, elle découle très exactement de la volonté de puissance, qui est la volonté de vie. À supposer que cette théorie soit nouvelle, cette réalité est le fait premier de toute histoire : ayons donc l'honnêteté de le reconnaître !18

On peut émettre l'hypothèse d'une réminiscence de Nietzsche chez Marinetti, tout comme pour la seconde Considération Inactuelle, «De l'utilité et de l'inconvénient des études historiques pour la vie ", et sa fortune dans l'élaboration de la notion de passatismo : mais Marinetti délaissera cette fois tout approfondissement pour s'en tenir à la guerre comme grande épreuve nationale et futuriste. Ce choix rappelle celui du manifeste La nouvelle religion-morale de la vitesse de l'année suivante, dans lequel Marinetti aboutit à une polémologie cosmique ("Participons donc aux grandes batailles célestes [...] ces guerres où les astres à la fois projectiles et artilleurs luttent de vitesse..." ${ }^{19}$ ) qu'il choisit à nouveau de ne pas approfondir, pour s'en tenir cette fois à une hypostase répétée de la Vitesse.

Ce sont là les deux uniques manifestes futuristes, sur quelques centaines, à énoncer clairement une polémologie de type héraclitéen ${ }^{20}$. Il faut donc nuancer la thèse d'une métaphysique polémologique du Futurisme: sans en nier la présence, on peut cependant constater ses limites et son hétéronomie par rapport à une ontologie de la vitesse (le dinamismo de matrice bergsonienne) et, plus fondamentalement encore, à cette éthique héroïque qui nous ramène enfin à Georges Sorel, dont l'influence dans les premières années du siècle était considérable en France comme en Italie.

Le volume Réflexions sur la violence publié en 1908 à Paris est constitué d'articles parus dans Il Divenire sociale, la revue romaine la plus en vue du syndicalisme révolutionnaire dirigée par Enrico Leone et publiée entre 1905 et 1910, puis repris dans Le mouvement socialiste, fondée à Paris en 1899 et publiée jusqu'en 1914 par Hubert Lagardelle et Jean Longuet. Il s'agit là de revues de "socialisme scientifique" qui étaient en réalité les organes $\mathrm{du}$ mouvement syndicaliste révolutionnaire que tentait de théoriser ce polytechnicien philosophe et sociologue franc tireur, pour lequel le syndicat est l'instrument de l'amélioration des conditions de vie du travailleur, mais aussi l'organe qui doit accoucher de la société future à travers la révolutio. À partir de ses lectures de 
Proudhon, de Marx, de Bergson, de Renan et de Péguy, mais également de Nietzsche, Sorel prône jusqu'en 1906, peu après la création de la SFIO et la charte d'Amiens, l'émancipation de la classe ouvrière par la violence et l'action directe. À partir de 1908, il se rapproche paradoxalement de Maurras, sans toutefois en partager entièrement le nationalisme, avant de saluer vivement la Révolution d'octobre, jugeant Lénine comme "le plus grand théoricien que le socialisme ait eu depuis Marx".

Sorel avait des liens personnels très forts avec l'Italie et de nombreux correspondants et amis italiens, de Pareto à Croce (qui préfaça l'édition italienne des Réflexions sur la violence). Il influença des hommes politiques de première importance comme Gramsci ou Mussolini par sa lecture de Marx fondée sur l'action directe et sur le rôle moteur du mythe dans l'histoire - en particulier celui de la grève générale -, ainsi que pour son exaltation de la violence, sa critique du libéralisme et du régime parlementaire et son individualisme libertaire, sans oublier sa haine envers l'État : ses volumes Saggi di critica del marxismo, paru à Palerme en 1903, et Lo sciopero generale e la violenza, édité par Mario Leone, et paru à Rome en 1906, avaient ainsi suscité de larges échos ${ }^{21}$.

Ses Réflexions sur la violence, parues en Italie en 1909, et dans lesquelles est précisément omniprésent le Nietzsche de Par delà Bien et $\mathrm{Mal}^{22}$, sont définies par Sorel lui-même comme une "apologie" et une "philosophie de la violence", qui deviendront l'un des aspects centraux de ce qu'on peut appeler l'antiphilosophie futuriste. Dans le cadre de la préparation à la grève générale, écrit Sorel,

Chaque conflit qui donne lieu à des violences devient ainsi un combat d'avantgarde, et personne ne saurait prévoir ce qui peut sortir de tels engagements; la grande bataille a beau fuir : en l'espèce, chaque fois qu'on en vient aux mains, c'est la grande bataille napoléonienne (celle qui écrase définitivement les vaincus) que les grévistes espèrent voir commencer; ainsi s'engendre, par la pratique des grèves, la notion d'une révolution catastrophique. [...]

La grève apporte une clarté nouvelle; elle sépare, mieux que les circonstances journalières de la vie, les intérêts et les manières de penser des deux groupes de salariés (le groupe administratif et le prolétariat) [...] la société est bien divisée en deux camps, et seulement deux, sur un champ de bataille. [...]

Plus de paix sociale possible, plus de routine résignée, plus d'enthousiasme pour les maîtres bienfaisants ou glorieux, le jour où les plus minimes incidents de la vie journalière deviennent des symptômes de l'état de lutte entre les classes, où tout conflit est un incident de guerre sociale, où toute grève engendre la perspective d'une catastrophe sociale. L'idée de grève générale est à ce point motrice qu'elle entraîne dans le sillage révolutionnaire tout ce qu'elle touche. ${ }^{23}$

La grève générale, la clarté nouvelle qu'elle induit dans une division nette entre deux camps distincts, et deux seulement, sa fonction résolutive et sa dimension catastrophique semblent préfigurer nettement 1915 In quest'anno futurista et la constitution du Futurisme comme réaction au passéisme, ainsi que sa capacité à polariser la société italienne jusqu'au conflit salvateur qui divise "partout et à tout propos, dans les parlements, les conseils municipaux et sur les places", les hommes "en passéistes et futuristes".

27 Il semble donc probable que les théoriciens du mouvement - Marinetti, Boccioni et Papini notamment 羊 aient trouvé là une source inspiratrice importante à la fois quant à leur rhétorique polémologique et à leur volonté révolutionnaire, en substituant le prolétariat, dont Sorel faisait le seul utilisateur légitime de la violence d'un point de vue historique, par une classe inédite et inouïe : "nous, les jeunes et forts futuristes".

Le Futurisme comme mythe sorélien 
Si l'analogie est frappante entre les lignes de Sorel et le projet de l'avant-garde futuriste, nous allons revenir maintenant sur un second aspect de l'influence sorélienne sur Marinetti, à savoir l'équivalence entre le mythe de la grève générale pour la révolution prolétaire, tel que Sorel le définit, et le projet d'ensemble du Futurisme. Sorel lui-même évoque le mythe comme

un ensemble d'images de batailles assurant le triomphe de leur cause [...] la grève générale des syndicalistes et la révolution catastrophique de Marx sont de tels mythes, [tout comme] ceux construits par le christianisme primitif, par la Réforme, par la Révolution, par les mazziniens [...] il faut les prendre en bloc de tels systèmes d'images, comme des forces historiques. ${ }^{24}$

Chez Sorel comme chez le "mazzinien" Marinetti ${ }^{25}$, la "révolution catastrophique" prônée est d'abord la "destruction rapide" d'une société que le capitalisme ou le passéisme ont longuement bâtie et structurée, et cette révolution politique est inséparable d'un engagement éthique qui touche à la "vie journalière". Cette interprétation éthique et politique de la polémologie marinettienne semble d'ailleurs plausible si l'on considère une autre équivalence arithmétique posée de façon bien plus ferme dans 1915 In quest'anno futurista : "Guerre = Futurisme intensifié", très fortement induite par la rhétorique et par les déterminations respectives caractérisant les deux termes (on a pu constater à quel point Guerra et Futurismo sont interchangeables dans le dernier paragraphe cité) ${ }^{26}$. C'est cette dernière égalité qui donne enfin sa cohérence esthétique, éthique et politique à l'ensemble du manifeste. 1915 In quest'anno futurista peut être lu comme la tentative la plus aboutie de définir le Futurisme comme un mouvement guerrier, et la guerre comme principe même de "l'évolution incessante et indéfinie" des individus et des nations, que Marinetti appellera dans d'autres textes le "Devenir fou".

30 Il semble utile de revenir alors sur le rôle moteur du mythe dans l'Histoire chez Sorel, en particulier celui de la grève générale, afin de le rapprocher à ce qui a été dit sur le mot magique Futurismo: le mythe et l'utopie chez Sorel ont en effet un sens qu'il peut être utile de rappeler ici.

Les mythes révolutionnaires actuels sont presque purs [...] ce ne sont pas des descriptions de choses, mais des expressions de volontés. L'utopie est au contraire le produit d'un travail intellectuel; elle est l'œuvre de théoriciens qui [...] cherchent à établir un modèle auquel on puisse comparer les sociétés existantes pour mesurer le bien et le mal qu'elles renferment [...] tandis que nos mythes actuels conduisent les hommes à se préparer à un combat pour détruire ce qui existe, l'utopie a toujours eu pour effet de diriger les esprits vers des réformes qui pourront êtres effectuées en morcelant le système; [...] un mythe ne saurait être réfuté puisqu'il est, au fond, identique aux convictions d'un groupe, qu'il est l'expression de ces convictions en langage de mouvement, et que, par suite, il est indécomposable en parties. ${ }^{27}$

31 Cette opposition entre le mythe et l'utopie peut éclairer des textes futuristes comme 1915 In quest'anno futurista, ou encore Al di là del Comunismo ${ }^{28}$, opuscule d'abord publié par la revue La Testa di Ferro à Milan, en 1919, puis réédité en 1924 et 1929 : bien qu'il s'agisse avec Le Tactilisme de l'un des textes les moins bellicistes de Marinetti, ces deux manifestes sont eux aussi et encore une fois tout entiers voués à stimuler une volonté révolutionnaire et violente, bien plus qu'à établir des modèles idéaux utiles à une réforme sociale. Il est vrai que dans le cas précis de ces deux manifestes, la préparation au "combat pour détruire ce qui existe" est bien moins prégnante que dans les textes qui précèdent la Grande Guerre, tels que 1915 In quest'anno futurista ou même, dès 1911, 
La guerra elettrica. De ce point de vue, le fléchissement du bellicisme futuriste après le traumatisme de la première guerre mondiale est indéniable, quoique les textes des années Trente renouent avec un propagandisme impérialiste inquiétant. Il semble toutefois exister une différence de nature entre les manifestes évoqués ici et ces textes tardifs fustigés par Benjamin : seuls les premiers, en effet, relèvent tous de la catégorie du mythe révolutionnaire que Sorel a tenté de définir à propos de la grève générale.

Il faut juger les mythes comme des moyens d'agir sur le présent [...] c'est l'ensemble du mythe qui importe seul [...]

Grâce à eux [les hommes qui prennent une part très active au mouvement révolutionnaire], nous savons que la grève générale est bien ce que j'ai dit: le mythe dans lequel le socialisme s'enferme tout entier, c'est-à-dire une organisation d'images capables d'évoquer instinctivement tous les sentiments qui correspondent aux diverses manifestations de la guerre envisagée par le socialisme contre la société moderne. [...] Nous obtenons ainsi une intuition du socialisme que le langage ne pouvait pas donner d'une manière parfaitement claire - et nous l'obtenons dans un ensemble perçu instantanément. ${ }^{29}$

Comme pour tout ce qui relève de la violence et dont il a été fait mention plus haut, il est possible de filtrer les propos de Sorel sur le socialisme afin de retrouver la définition opératoire du Futurisme telle que Marinetti la présente dans des manifestes aussi éloignés que 1915 In quest'anno futurista et Al di là del Comunismo : une organisation d'images perçue instantanément et quasi indicible, capable d'évoquer instinctivement en chacun - en chaque jeune étudiant et artiste italien de génie, voire dans le "prolétariat des génies" et dans "l'artécratie" de 1919 - l'ensemble des sentiments guerriers contre une société pourrie de passéisme. Cette intuition de type bergsonien de ce que doit être la grève générale, selon les mots même de Sorel, c'est précisément ce qu'a tenté de constituer le Futurisme au travers d'une accumulation de récits et de spectacles (dont par exemple "ce phénomène qu'est la Soirée futuriste (propagande très efficace de courage)" évoquée supra), de lois et de médias, enfin d'œuvres artistiques littéraires, musicales, plastiques et d'autres encore, "images de batailles assurant le triomphe de leur cause" qu'il faut "prendre en bloc, comme des forces historiques", pour rappeler les propos de Sorel cités ci-dessus ${ }^{30}$. Marinetti fera ainsi du Futurisme même une intuition mythique, un système d'images autoréférentiel, et une incantation enthousiaste, épique et révolutionnaire, incommensurable à tout autre système philosophique.

з3 Ces remarques sur la proximité entre Sorel et le Futurisme mériteraient quelque approfondissement : ainsi, l'examen rapide des rares citations directes de Sorel par Marinetti est également révélateur de la volonté futuriste de se clore en un système d'image saisissable intuitivement et sans analyse rationnelle.

Si les réflexions soréliennes sur la violence transparaissent jusque dans le titre de la conférence de Marinetti Necessità e bellezza della violenza, prononcée dans plusieurs villes italiennes et dans des cadres syndicalistes et socialistes en 1910, Marinetti prend bien plus de distances dans un article de 1919, Crollo di filosofi e storici, sibille a rovescio, publié in Democrazia futurista, puis Futurismo e fascismo, pour adopter face à Sorel le même type de posture qu'il avait réservée à Nietzsche dans Contro $i$ professori ${ }^{31}$ en 1915. Il s'agit alors d'un reniement radical et sans appel, qui sera la marque des avant-gardes face à leurs pères et à leurs généalogies ${ }^{32}$. Luciano de Maria - apologie de la violence, antiparlementarisme, exaltation d'une 
morale individuelle hérö̈que induite sans doute de Péguy, critique violente de la bourgeoisie et de l'Etat ${ }^{33}$ - demeurent eux-mêmes tributaires, le plus souvent, d'une médiation et d'une synthèse sorélienne d'autres penseurs : Péguy donc, mais tout aussi bien Renan, Marx, et surtout Bergson et Nietzsche. Concernant Nietzsche, du reste, il serait encore aisé de vérifier que la distinction sorélienne entre "morale des producteurs" et "morale des consommateurs", que Marinetti transpose dans tous ses textes poétiques en s'en tenant délibérément au point de vue des créateurs plutôt qu'à celui du public, dérive explicitement de la lecture nietzschéenne des chapitres de Par delà Bien et Mal et de la Généalogie de la Morale consacrés à la morale des maîtres et à celle des esclaves ${ }^{34}$.

Enfin, par-delà la confirmation et l'identification plus précise d'une influence de Sorel sur Marinetti et de la médiation partielle de Sorel dans la lecture marinettienne de Nietzsche, cette généalogie nous a semblé mettre à jour des types d'intertextualités originales, irréductibles à la citation, à la référence, à l'allusion ou au plagiat relevés par Genette ${ }^{35}$, mais plutôt inhérentes à la prose d'idée à l'ère de la reproductibilité technique et de la société de masse: une intertextualité qu'on pourrait qualifier d'opératoire (le Futurisme marinettien comme mythe sorélien actualisé et autoréférentiel) et des intertextualités de type analogique (violence prolétarienne contre la bourgeoisie comme classe chez Sorel - violence des jeunes contre le passéisme comme maladie individuelle, culturelle et nationale chez Marinetti; grève générale comme force historique chez Sorel - Futurisme comme force incantatoire chez Marinetti; morale des aristocrates, des producteurs et des créateurs - morale des esclaves, des consommateurs et des spectateurs respectivement chez Nietzsche, Sorel et Marinetti).

Il pourrait être utile de revenir dès lors sur quelques créations futuristes afin de vérifier comment pour chacune d'entre elles - plastique, littéraire, musicale ou hybride qu'elle puisse être, ces intertextualités propagandistes contribuent à constituer l'œuvre entre art, manifeste et produit de consommation d'une part, mais aussi entre désinvolture, dénégation et subversion sérieuse d'autre part - l'intertextualité mélancolique et passéiste n'étant bien sûr jamais une option permise explicitement à l'avant-garde.

\section{NOTES}

1. Cf. par exemple les écrits de Luciano De Maria, Pär Bergman, Roberto Tessari, Enrico Crispolti, Luciano Caruso, Claudia Salaris ou Giovanni Lista, pour ne citer que les plus connus, et sans compter quelques contributions des contemporains au mouvement comme Benedetto Croce ou Auguste Joly.

2. L'Italia futurista, Florence, $\mathrm{n}^{\circ} 15$ et 18,27 mai et 17 juin 1917. Cf. F.T. MARINETTI, Teoria e invenzione futurista, "I Meridiani”, Arnoldo Mondadori editore, Milan, 1968. 
3. F.T. MARINETTI, "Le futurisme", in Le Figaro, Paris, 20 février 1909, et Primo manifesto politico futurista per le elezioni generali del 1909, Milan, Direction du mouvement futuriste, 1909.

4. F.T. MARINETTI, ibid., et Uccidiamo il Chiaro di Luna !, in Poesia, a. V, n 7, 8, 9, Milan, août/ septembre/octobre 1909.

5. "Place aux jeunes!". Cf. U. BOCCIONI, C. CARRÀ, L. RUSSOLO, G. BALLA, G. SEVERINI, Manifesto dei pittori futuristi, Direction du mouvement futuriste, 1910.

6. Cf. A. SANT'ELIA, L'architettura futurista, Milan, Direction du mouvement futuriste, 1914, et F.T. MARINETTI, FILLİA, La cucina futurista, Milan, Sonzogno, 1932.

7. F.T. MARINETTI, 1915 In quest'anno futurista, in Guerra sola igiene del mondo, Milan, Edizioni futuriste di Poesia, 1915. Les traductions des textes cités sont parfois des versions françaises rédigées par Marinetti lui-même, ou sont empruntées en partie à G. LISTA, Futurisme - Manifestes, Proclamations, Documents, Lausanne, L'Age d'Homme, 1973.

8. Il faut rappeler la volonté provocatrice d'un volume et d'un titre comme Guerra sola igiene del mondo, traduction italienne du recueil publié quatre ans plus tôt à Paris pour Sansot, Le futurisme, et remanié par Marinetti dans un but propagandiste explicite ("scopo propaganda" ["but propagande"] avait-il fait écrire sur le frontispice).

9. Ibid. Nous avons taché de rendre la "typographie expressive" des textes originaux. 10. Plus précisément, il s'agit des deux premiers paragraphes de l'analyse qui suit, qu'on retrouvera in Lettera aperta al futurista Mac Delmarle, in Lacerba, a. I, n 16, Florence, 15 août 1913, et Democrazia futurista - Dinamismo politico, Milan, Facchi, 1919 (premier chapitre).

11. Cf. Il carteggio Carrà-Papini - Documenti del MART, édité par Massimo Carrà, Milan, Skira 2001. Papini écrit à Carrà le 22 janvier 1915 : "... Marinetti [...] fait des allusions accusatrices contre moi et Lacerba dans son dernier manifeste." On a la confirmation qu'il s'agit bien de 1915 In quest'anno futurista en lisant la réponse de Carrà du 28 janvier, qui évoque bien "le manifeste aux étudiants".

12. F.T. MARINETTI, 1915 In quest'anno futurista, in Guerra sola igiene del mondo, cit., 1915.

13. Cf. F.T. MARINETT, Prime battaglie futuriste, Le battaglie di Trieste, ou Movimento politico futurista, in Guerra sola igiene del mondo, cit., 1915. On constatera cette même revendication dans les premiers chapitres de Democrazia futurista, cit., 1919, ainsi que dans Futurismo e Fascismo, cit., 1924.

14. F.T. MARINETTI, Uccidiamo il Chiaro di Luna !, cit., 1909.

15. F.T. MARINETTI, 1915 In quest'anno futurista, in Guerra sola igiene del mondo, cit., 1915.

16. Cf. « Le Futurisme italien et la question des valeurs ", in Noésis, $n^{\circ} 11$, Art et Politique, CRHI, Nice, à paraître en ligne sur. http://revel.unice.fr/noesis/

17. F.T. MARINETTI, 1915 In quest'anno futurista, in Guerra sola igiene del mondo, cit., 1915.

18. F. NIETZSCHE, Par delà Bien et Mal, $\S 259$ (trad. C. Heim, in Euvres philosophiques complètes, textes et variantes établis par G. Colli et M. Montinari, Paris, Gallimard Nrf, Tome VII, p. 182).

19. F.T. MARINETTI, La nuova religione-morale della velocità, Direction du mouvement futuriste, 1916.

20. Pour Polemos comme père et roi de toute chose, cf. HÉRACLITE, fragment LXXX.

21. G. SOREL publia ensuite en Italie Insegnamenti sociali della economia contemporanea.

Degenerazione capitalista e degenerazione socialista, Palerme, Sandron, 1906, et

Considerazioni sulla violenza, préface de Benedetto Croce, Bari, Laterza, 1909. Pour une

bibliographie de Sorel en Italie, cf. G.B. FURIOzzI, "Sorel e l'Italia. Bibliografia ragionata", 
in Annali della facoltà di scienze politiche, Pérouse, 1970, pp. 173-206, et G. SOREL, Scritti politici e filosofici, Einaudi, Turin, 1975, pp. LIII-LXXI (bibliographie de G. Cavallari). 22. G. SOREL, Réflexions sur la violence, Paris, Pages libres, 1908 (réédition Paris, Seuil, 1990 , p. 266 et suivantes).

23. Ibid. , pp. 81 et 147.

24. Ibid., p. 36.

25. Pour Marinetti en tant que "mazzinien" tardif, on se souviendra par exemple de sa table mot-libriste Irredentismo de 1912, de ses citations mazziniennes en forme de slogans ("Le mot Italie doit primer sur le mot liberté"), et des articles qu'il consacra à Mazzini, par exemple in Democrazia futurista (dinamismo politico), cit., 1919.

26. MARINETTI la reprendra la même année dans un autre manifeste écrit avec Emilio SETTIMELLI et Bruno CORRA, Il Teatro futurista sintetico, Direction du mouvement futuriste, 1915.

27. G. SOREL, op. cit., p. 46.

28. F.T. MARINETTI, Futurismo e fascismo, Milan, Franco Campitelli editore, 1924, et Marinetti e il futurismo, Rome-Milan, Augustea, 1929. Le texte a paru également dans plusieurs revues futuristes.

29. Ibid., p.141.

30. Ibid., p. 36.

31. Ce texte est lui-même une reprise propagandiste et provocatrice de l'un des chapitres du volume Le futurisme, 1911, op. cit., intitulé plus sobrement «Ce qui nous sépare de Nietzsche ».

32. Cf. " Nous renions nos maîtres symbolistes, derniers amants de la lune ", in Le futurisme, op. cit. Marinetti, Tzara, Breton, Debord subiront à leur tour ce même sort de la part de leurs épigones, sort prophétisé dans la partie finale du manifeste de fondation marinettien.

33. L. DE MARIA, «Marinetti poeta e ideologo», introduction à F.T. Marinetti, Teoria e invenzione futurista, Milan, «Meridiani», Arnoldo Mondadori, 1968, p. LVII.

34. Cf. G. SOREL, op. cit., Paris, Seuil, 1990, p. 266 - 283.

35. G. GENETTE, Palimpsestes - La littérature au second degré, Paris, Seuil, 1982.

\section{RÉSUMÉS}

Cet article se propose de revenir sur la filiation idéologique largement établie entre certains écrits de Georges Sorel, et notamment ses Réflexions sur la violence publiées en 1909, et les manifestes du Futurisme italien, la première avant-garde pluridisciplinaire européenne s'étant constituée autour d'un projet à la fois esthétique, politique et anthropologique. En particulier, il s'agit de revenir sur les emprunts idéologiques de Filippo Tommaso Marinetti aux écrits de Sorel dans des manifestes d'importance tels que 1915 En cette année futuriste ou Par-delà le Communisme (1919), et de revenir également sur le type d'intertextualité établi par ces textes au sein de la prose d'idée. 
In this article, we will touch upon the ideological filiation introduced by some of Georges Sorel writings - and in particular Réflexions sur la violence published in 1909 - and the manifests of italian Futurism, the first european multidisciplinary avant-garde project to include an aesthetic, political and anthropological view. In particular, we will examine Sorel's ideological influence on Filippo Tommaso Marinetti's 1915 In quest'anno futurista and Al di là del Comunismo, and we will look back on the intertextuality produced in those texts inside the prose of ideas.

Quest'articolo propone di rivisitare la filiazione ideologica già stabilita tra gli scritti di Georges Sorel, e più precisamente le sue Réflexions sur la violence publicate nel 1909, ed i manifesti del Futurismo italiano, prima avanguardia europea strutturatasi in un progetto tanto estetico e politico quanto più generalmente antropologico. In particolare, sono sottolineati i prestiti sorelliani a Filippo Tommaso Marinetti in manifesti quali 1915 In quest'anno futurista o Al di là del Comunismo, ed i tipi di intertestualità stabiliti da questi testi all'interno della prosa di idee.

INDEX

Mots-clés : idéologie, avant-garde, Futurisme, Nietzsche, Georges Sorel, Marinetti

\section{AUTEUR}

\section{SERGE MILAN}

Université de Nice-Sophia Antipolis, CIRCPLES, EA 3159 